\title{
Evaluation of the Diagnostic Value of Extra-criteria Antibodies for Antiphospholipid Syndrome Patients in a Chinese Cohort
}

\author{
Chaojun $\mathrm{Hu}^{1}$, Siting $\mathrm{Li}^{1}$, Zhijuan $\mathrm{Xie}^{1}$, Hanxiao You ${ }^{1}$, Hui Jiang ${ }^{1}$, Yu Shi ${ }^{1}$, Wanting $\mathrm{Qi}^{1}$, \\ Jiuliang Zhao ${ }^{1}$, Qian Wang ${ }^{1}$, Xinping Tian ${ }^{1}$, Mengtao $\mathrm{Li}^{1}$, Yan Zhao ${ }^{1}$, and Xiaofeng Zeng ${ }^{1}$ \\ ${ }^{1}$ Peking Union Medical College Hospital
}

April 24, 2021

\begin{abstract}
Objective: Although specific anti-phospholipids antibodies (aPLs) have been used in the diagnosis of the antiphospholipid syndrome (APS) for years, new biomarkers are required to increase its diagnostic as well as risk-predictive power. This study aimed to explore the value of several extra-criteria aPLs in a Chinese cohort. Methods: A total of 312 patients including 100 patients diagnosed with primary APS, 51 with APS secondary to SLE, 71 with SLE, and 90 health controls were recruited. Serum anticardiolipin (aCL) IgG/IgM/IgA, anti-ß2-glycoprotein I (aß2GPI) IgG/IgM/IgA, anti-phosphatidylserine/prothrombin antibodies (aPS/PT) IgG/IgM, anti-annexin A5 antibodies (aAnxV) IgG/IgM were tested using ELISA kits. Results: Totally $30.46 \%$ and $6.62 \%$ of patients with APS were positive for aCL or a $32 \mathrm{GPI}$ IgA respectively, while $39.07 \%$ and $24.50 \%$ were positive for aAnxV or aPS/PT for at least one antibodies (IgG or IgM). The addition test of aCL IgA and aAnxV IgM assist in identifying seronegative APS patients, and IgG aANxV was linked with stroke. Conclusion: Detection of aCL IgA, aß2GPI IgA, $\mathrm{aAnxV} \operatorname{IgG} / \mathrm{M}$, and aPS/PT IgG/M as biomarker provide additive value in APS diagnosis, and would help in risk prediction for APS patients in medical practice.
\end{abstract}

\section{Evaluation of the Diagnostic Value of Extra-criteria Antibodies for Antiphospholipid Syndrome Patients in a Chinese Cohort}

Chaojun $\mathrm{Hu}^{1,2, \#}$, Siting $\mathrm{Li}^{1,2}, \#$, Zhijuan $\mathrm{Xie}^{1,2}$, Hanxiao You ${ }^{1,2}$, Hui Jiang ${ }^{1,2}$, Yu Shi ${ }^{1,2}$, Wanting Qi ${ }^{1,2}$, Jiuliang Zhao ${ }^{1,2, *}$, Qian Wang ${ }^{1,2}$,Xinping Tian ${ }^{1,2}$, Mengtao Li $^{1,2}$, Yan Zhao ${ }^{1,2}$,Xiaofeng Zeng ${ }^{1,2, *}$

${ }^{1}$ Department of Rheumatology, Peking Union Medical College Hospital, Peking Union Medical College \& Chinese Academy of Medical Sciences; Key Laboratory of Rheumatology \& Clinical Immunology, Ministry of Education, Beijing, 100730, China;

2 National Clinical Research Center for Dermatologic and Immunologic Diseases (NCRC-DID), Beijing, 100730, China;

\# Chaojun $\mathrm{Hu}$ and Siting Li contributed equally to this work and should be considered as co-first authors.

*Correspondence to: Jiuliang Zhao, Department of Rheumatology, Peking Union Medical College Hospital, Peking Union Medical College \& Chinese Academy of Medical Sciences; Key Laboratory of Rheumatology \& Clinical Immunology, Ministry of Education, No. 1 Shuaifuyuan, Beijing, 100730, China. E-mail:

zjlpumc@sina.com; Xiaofeng Zeng, Department of Rheumatology, National Clinical Research Center for Immunological Diseases, Peking Union Medical College 83 Chinese Academy of Medical Science, Key Laboratory of Rheumatology and Clinical Immunology, Ministry of Education, Beijing 100730, China. Email: zengxfpumc@163.com.

Abstract 
Objective:Although specific anti-phospholipids antibodies (aPLs) have been used in the diagnosis of the antiphospholipid syndrome (APS) for years, new biomarkers are required to increase its diagnostic as well as risk-predictive power. This study aimed to explore the value of several extra-criteria aPLs in a Chinese cohort.

Methods: A total of 312 patients including 100 patients diagnosed with primary APS, 51 with APS secondary to SLE, 71 with SLE, and 90 health controls were recruited. Serum anticardiolipin (aCL) IgG/IgM/IgA, anti- $\beta 2$-glycoprotein I (aß2GPI) IgG/IgM/IgA, anti-phosphatidylserine/prothrombin antibodies (aPS/PT) IgG/IgM, anti-annexin A5 antibodies (aAnxV) IgG/IgM were tested using ELISA kits.

Results: Totally $30.46 \%$ and $6.62 \%$ of patients with APS were positive for aCL or aß2GPI IgA respectively, while $39.07 \%$ and $24.50 \%$ were positive for aAnxV or aPS/PT for at least one antibodies (IgG or IgM). The addition test of aCL IgA and aAnxV IgM assist in identifying seronegative APS patients, and IgG aANxV was linked with stroke.

Conclusion: Detection of aCL IgA, aß2GPI IgA, aAnxV IgG/M, and aPS/PT IgG/M as biomarker provide additive value in APS diagnosis, and would help in risk prediction for APS patients in medical practice.

Keywords antiphospholipid syndrome, antiphospholipid antibodies, immunoglobulin A, antiphosphatidylserine/prothrombin, anti-annexin V

\section{Introduction}

The antiphospholipid syndrome (APS) is a systemic autoimmune disease characterized by thrombosis and/or pregnancy morbidity with the persistent presence of medium or high titer of antiphospholipid antibodies (aPLs). The golden standard for APS diagnosis is the 2006 APS classification criteria (Sydney criteria), where at least one of the clinical criteria, as well as one of laboratory criteria including lupus anticoagulant (LA), high level of anti-cardiolipin (aCL), anti- $\beta 2$ glycoprotein-I (aß2GPI) immunoglobulin isotype G (IgG) or M (IgM), should be present(1). Despite its wide use in clinical practice, patients could remain persistently negative for criteria aPLs yet show typical APS clinical manifestations (defined as seronegative APS, SNAPS(2)), and a broader range of diagnostic biomarkers are required (3). Apart from standard criteria, other extra-criteria clinical and laboratory features have been found associated with APS in numerous studies, which involves heart valve disease, thrombocytopenia, neurological manifestations, anti-CL or anti32GpI IgA, anti-phosphatidylserine-prothrombin (aPS/PT) complex, anti-annexin A5 antibodies (aAnxV), etc $(4,5)$. Besides APS diagnosis, evaluation of non-criteria aPLs could also contribute to prognosis and risk assessment for associated clinical manifestations(6).

More specifically, numerous studies had been conducted to investigate the diagnostic value of aCL/aß2GpI IgA for APS, which received contradictory results(7). Nevertheless, testing of IgA had been recommended by guidelines when criterial aPLs remained negative(8). In addition, aAnxV and aPS/PT are receiving continuous attention in recent years. AnxV is a phospholipid-binding protein highly expressed in vascular endothelial cells. It could bind tightly to exposed anionic phospholipids and assemble into a shield, which may prevent phospholipid-dependent coagulation reaction $(9,10)$. In a systemic review, AnxV resistance has been observed and analyzed to have a higher prevalence in APS compared to disease controls(11) and has been reported to be linked with hypercoagulable state as well as obstetric complications in APS patients $(12,13)$. Furthermore, its anticoagulant activity was reduced by plasmas of patients with APS and thromboembolism(14), and loss of maternal aAnxV increased the chance of placental platelet thrombosis and fetal loss(15). However, other studies found no significant association between thrombotic event or adverse pregnancy manifestations $(16,17)$.

Prothrombin is another phospholipid-binding protein which forms complex and is often co-detected of antibodies together with phosphatidylserine (aPS/PT). An international multi-centre study confirmed the contribution of aPS/PT IgG in APS diagnosis IgG(18). Concerning its relation with clinical features such as thrombotic events or obstetric complications, conflicting results had been shown and confirmation is still needed $(19,20)$. Nevertheless, numerous studies have indicated a strong correlation between aPS/PT and 
$\mathrm{LA}(21,22)$. In addition, a higher level of aPS/PT was observed to be associated with high-risk "triple positive" patients (LA+, aCL IgG and/or IgM +, and aß2GPI IgG and/or IgM+)(23), and may also add value to identification of SNAPS(3).

Studies design, including detection method, patient stratification, population heterogeneity, etc., may lead to contradictory results in different studies. Regarding the Chinese population, a previous study indicated an increase of both IgG and IgM aAnxV in primary APS patients and APS associated with other diseases. Significant associations were also observed between IgG aANxV and thrombotic events(24). Additionally, assessment of the diagnostic performance of aPS/PT revealed a significant correlation between thrombotic events and pregnancy loss with $\operatorname{IgG}$ aPS/PT $(25,26)$, which was confirmed by a recent study $(27)$. Concerning aCL/aß2GpI IgA, a study recently conducted by us in a large Chinese population revealed little added diagnostic value(28). Few studies have explored all of the above extra-criteria autoantibodies in the same patient groups, and their relations with more detailed clinical manifestations still need investigation. This study focused on evaluating the additive diagnostic value of aCL/aß2GpI IgA, IgG and IgM for aANxV or aPS/PT to standard aPLs in a Chinese cohort. Correlation with clinical features including thrombotic events, obstetric complications, as well as microangiopathy was also explored.

\section{Patients and methods}

\section{Patients recruitment}

This was a single-center, prospective cohort study conducted at Peking Union Medical College Hospital (PUMCH) from May 2017 to January 2020. A total of 312 consecutive patients were included in this study, of which 100 patients had been diagnosed with primary APS (PAPS group), 51 with APS secondary to SLE (SAPS group), 71 with SLE (SLE group), and 90 health controls (HC group). Diagnosis of APS was defined by clinicians according to the 2006 Sydney revised classification criteria. Sera samples were collected and immediately profiled of aPL antibodies at the Key Laboratory of Department of Rheumatology, Peking Union Medical College Hospital (PUMCH). Besides aPL serology, clinical manifestations were recorded for PAPS, SAPS, and SLE groups, including thrombosis (arterial or venous), pregnancy morbidity, microangiography (deep venous thrombosis, pulmonary embolism, etc.), and history of adverse pregnancy. For HC group, only aPL serology information was present. The study was approved by ethics committee at PUMCH and fulfilled the ethical guidelines of the declaration of Helsinki. All subjects gave written informed consent.

\section{Laboratory tests}

IgG, IgM, and IgA isotypes of aCL and aß2GPI, IgG and IgM isotypes of aPS/PT and aAnxV were analyzed with AESKULISA $\cap$ ELISA Test Kits provided by Aesku. Diagnostics GmbH \& Co. KG (Wendelsheim, Germany). Cut-off value was defined as $18 \mathrm{U} / \mathrm{mL}$ as recommended by the manufacturer. Lupus anticoagulant was detected and evaluated at the Key Laboratory according to the ISTH recommendations measuring Dilute Russell viper venom time (dRVVT)/activated partial thromboplastin time ( $>1.20$ as positive).

\section{Statistical analysis}

Statistical analysis was performed using SPSS 26.0 or R (version 3.6.2). The $\chi^{2}$ test or Fisher's exact test was used for comparison of categorical variables, and Wilcoxon test was used for continuous variables after normality was explored with the Shapiro-Wilk test. Sensitivities, specificities, and accuracies in APS diagnosis were compared in the McNemar test. Youden Index, positive and negative predictive values (PPV and NPV), and odds ratio (OR) with $95 \%$ confidence interval (95\% CI) were also shown. Receiver operating characteristic (ROC) curves of individual aPL as well as logistic regression analysis of aPLs profile were used to calculate the area under the curve (AUC), with 95\% CI shown. Associations between aPL isotype positivity and clinical manifestation in patients with APS were explored and displayed in $95 \%$ CI. Two-tailed values of $P$ less than 0.05 were considered statistically significant.

\section{Results}

\section{Patient characteristics}


Among 151 APS patients, there were 63(63.0\%) females for PAPS, 45(88.2\%) for SAPS, and the mean age for each were 36.3 and 32.9 years (Table 1). The mean age was 30.1 years in SLE group, of which $61(85.9 \%)$ were female, while HC group had 41(45.6\%) female and a mean age of 43.4. Clinical manifestations were recorded for both APS and SLE patients and were selectively shown. Thrombosis was most commonly present, with $80(80.0 \%)$ for PAPS and $74.5 \%$ for SAPS, but not in SLE group. Patients were recorded for history of arterial or venous thrombotic events, pregnancy morbidity, microangiopathy, history of adverse pregnancy, and LA. Of all the clinical manifestations, prevalence of adverse pregnancy history was significant different between PAPS and SAPS group $\left(\chi^{2}=3.922, P=0.048\right)$.

\section{Predictive power of aPLs in APS diagnosis}

The diagnostic power of aPLs positivity $(>18 \mathrm{U} / \mathrm{ml}$ ) was evaluated for sensitivity, specificity, accuracy, Youden Index, PPV, NPV, and ORs in APS diagnosis from HC group in table 2. For IgA, the sensitivity and accuracy of the combination of aCL IgG, IgM, or IgA were significantly higher than that of aCL IgG or IgM $(\mathrm{p}<0.001)$, while specificity was lower $(\mathrm{p}=0.031)$. A similar result was observed for aCL or aB2GpI IgG or IgM or IgA compared to aCL or aB2GpI IgG or IgM. As for aAnxV, the sensitivity and accuracy of aAnxV IgG or IgM was significantly higher than that of aB2GpI IgG or IgM $(\mathrm{P}<0.001)$. In addition, a combination of aCL, a $\beta 2 \mathrm{GpI}$, or aAnxV IgG or IgM had significantly higher sensitivity $(\mathrm{p}=0.016)$ compared to that of aCL or a $32 \mathrm{GpI}$ IgG or IgM.

As illustrated in figure 1, ROC curves were applied to evaluate the predictive value of aPLs or their combined positivity. Among individual aPLs, aß2GP1 IgG (0.915), aCL IgA (0.853), aCL IgM (0.767), and aAnxV IgG (0.728) had the largest AUC values. Adding IgA, aAnxV or aPS/PT IgG or IgM to aCL or a $32 \mathrm{GpI} \operatorname{IgG}$ or IgM would both increase AUC (0.927, 0.951, and 0.936 compared to 0.925).

\section{Cross-positivity analysis for four aPLs in APS patients}

Among 151 APS patients, cross positivity of IgG, IgM, or IgA for aCL or a $32 \mathrm{GpI}$ (a and b), as well as IgG or IgM for each of the four aPLs (c and d) were demonstrated with Venn diagram in figure 2. For patients positive for aCL, 16 were positive only for IgA. Concerning IgG isotype, aCL and aAnxV IgG were most often positive among APS patients. As for IgM isotype, there were $12(7.9 \%)$ patients who were test positive only for aAnxV, and $4(2.6 \%)$ were positive only for aPS/PT.

The number of patients positive only for each of the five non-criterial aPLs was also calculated among SPAPS and SNAPS patients (not shown). Totally 12 patients for aCL IgA, 9 for aAnxV IgG, 5 for aAnxV IgM, 3 for aPS/PT IgM, 1 for aPS/PT IgG, and 1 for a $32 \mathrm{GpI}$ IgA were observed. On the other hand, among all 14 SNAPS patients, 7(50\%) were positive for extra criteria aPLs. ACL IgA and aAnxV IgM each identified 3 of these patients individually, while 1 patient was positive for aPS/PT IgM and a $\beta 2 \mathrm{GpI} \operatorname{IgA}$.

\section{Distribution of antiphospholipid antibodies}

The distribution of all criterial or non-criterial aPLs among different patient groups was shown in Figure 3. Levels of aPLs were calculated with $(\log ($ test value +2$) \mathrm{U} / \mathrm{ml})$. The results of primary or secondary APS were compared to other groups. No significant difference was observed between primary and secondary APS, except for aCL IgM $(p=0.029)$ and a $32 \mathrm{GpI} \operatorname{IgA}(\mathrm{p}=0.043)$. Compared to HC, levels of IgG and IgA were significantly higher for four aPLs in both PAPS and SAPS group. However, IgM results varied for different aPLs.

\section{Clinical manifestations of different aPLs in APS patients}

Correlations between different aPLs and LA or clinical manifestations were shown with odds ratios in table 3. Presence of LA was significantly associated with IgG of aCL (ORs 9.0, 95\% CI 2.6-31.0), a 32 GPI (ORs 14.1, 95\% CI 1.9-107.2), aPS/PT (ORs 4.7, 95\% CI 1.1-21.2), and aAnxV (ORs 21.5, 95\% CI 2.8-163.0). Among all microangiopathy manifestations, stroke was significantly associated with a 32 GPI IgG (ORs 4.7, 95\% CI 1.2-18.5) as well as aPS/PT IgG (ORs 6.5, 95\% CI 1.6-25.9). Additionally, aPS/PT IgM was reversely associated with pregnancy loss in women (ORs 0.6, 95\% CI 0.5-0.7). 


\section{Discussion}

APS is an autoimmune disease featuring thrombosis and/or pregnancy morbidity which may lead to severe consequences. Detection of aCL and a $32 \mathrm{GPI}$ as the golden standard in APS diagnosis is not satisfactory in the clinical scenario, and various potential aPLs have been extensively explored.

In this study, the diagnostic value of IgA for aCL or a $32 \mathrm{GPI}$, IgG/IgM for aANxV or aPS/PT was evaluated in APS patients. In brief, $45.70 \%$ and $6.62 \%$ of patients with APS were positive for aCL or aß2GPI IgA respectively, while $30.46 \%$ and $24.50 \%$ were positive for aAnxV or aPS/PT for at least one antibodies (IgG or IgM). Adding IgA to criterial aPLs could increase the sensitivity in APS diagnosis. Detection of aANxV or aPS/PT, especially aAnxV IgG, could add value to diagnosis. IgG of aANxV or aPS/PT was significantly associated with LA, and IgG aANxV was linked with stroke.

Analysis of the predictive power indicates that although aCL IgA had relatively low specificity, adding IgA to aCL IgG or IgM/aCL or a $32 \mathrm{GpI}$ IgG or IgM test could increase test sensitivity $(\mathrm{P}<0.001)$. The sensitivity (39.07\% compared to $29.14 \%, \mathrm{P}<0.001)$. and accuracy $(60.58 \%$ compared to $55.19 \%, \mathrm{P}=0.007)$ of aAnxV IgG or IgM were both significantly higher than that of a 32 GPI IgG or IgM. Moreover, combination of aCL, aß2GpI, or aAnxV IgG or IgM had significantly higher sensitivity (47.7\% compared to $43.0 \%, \mathrm{P}=0.016$ ) than that of aCL or a $32 \mathrm{GpI}$ IgG or IgM. Statistic results suggested that adding aAnxV IgG or IgM to aCL or a $32 \mathrm{GpI} \operatorname{IgG}$ or IgM would both increase diagnostic value besides criterial antibodies. Meanwhile, there was no significant decrease in specificity $(96.67 \%)$.

The result was further illustrated with ROC curves for each aPL and their combination. AUC of ACL IgA and aAnxV IgG ranked second and third (0.853 and 0.728) among individual aPLs. Addition of IgA, aAnxV or aPS/PT to aCL or a $\beta 2 \mathrm{GpI}$ IgG or IgM would all increase their diagnostic power.

Venn diagram indicated the additive value of new aPLs from another perspective. Positive only for IgA isotype could point out an extra number of patients for both aCL $(16,10.6 \%)$ and a $32 \mathrm{GPI}(4,2.6 \%)$. Additionally, the number of patients positive for aAnxV IgG, aAnxV IgM, and aPS/PT IgM outperformed those of a 32 GPI, indicating their importance in APS clinical diagnosis. The result suggested that additional tests for extra criteria aPLs could provide unique value in the identification of SNAPS patients.

Besides predictive power, distribution, and comparison of aPLs among different patient groups were also examined. Between PAPS and SAPS, little significant difference was observed except for aCL IgM $(\mathrm{p}=$ $0.029)$ and $a \beta 2 \mathrm{GpI} \operatorname{IgA}(\mathrm{p}=0.043)$. Between PAPS and SLE, significantly higher titer of IgM aCL, IgA aCL, IgM aPS/PT, IgG AnxV, and IgM AnxV was observed $(\mathrm{p}<0.001)$. As for SAPS and SLE, only IgM aPS/PT showed a significant difference $(\mathrm{p}=0.015)$. The results implied that both criterial and non-criterial aPLs had difficulty in distinguishing APS from SLE or APS secondary to SLE. Indeed, baseline information suggested little difference between PAPS and SAPS patients in age and most clinical manifestations (Table 1). It had been estimated in previous studies that around $40 \%$ of patients with SLE have aPL, and APS may develop in up to 50-70\% of patients with both SLE and aPL(29). Nevertheless, levels of IgG for four aPLs were significantly higher in both PAPS and SAPS group compared to HC, which suggested their diagnostic value.

Finally, the relationship between aPLs and related clinical manifestations was calculated. In this study, no significant association was found between aPLs with any thrombotic events, which was contradictory with results from some previous studies conducted in the Chinese population (24-26). Concerning obstetric complication, aPS/PT IgM was reversely associated with pregnancy loss in women (ORs 0.6, 95\% CI 0.5$0.7)$, which also showed conflicting results $(25,30,31)$. For aAnxV, similar to a previous study, no significant relationship was observed(24). The different results might be due to the detection system. ELISA was chosen in this study, and the cut-off value provided by the manufacturer $(18 \mathrm{U} / \mathrm{ml}$ for all the aPLs) may not reflect real aPL distribution in local population. Indeed, as illustrated in figure 2, 31 patients were negative for all IgG, while as many as 118 patients were negative for all IgM. It could be more suitable if 99th percentile strategy was adopted first to identify cut-off points for each aPLs. 
Additionally, the relationship between aAnxV and $\mathrm{aPS} / \mathrm{PT} \operatorname{IgG}$ and LA was confirmed in our studies, and LA was found to be associated with IgG of all four aPLs. Regarding microangiopathy, a series of manifestations had been recorded for the patients (stroke, deep venous thrombosis, pulmonary embolism, etc.), and significant relationship with aPLs (aß2GPI IgG and aPS/PT IgG) was present for stroke. Previous review has estimated an aPL positivity of $17 \%$ in patients with juvenile stroke ( $<50$ years of age) (32). Although detection of aPS/PT alone may have less diagnostic value, it would still be valuable in risk prediction for and prevention of adverse clinical events.

This study has some limitations. Compared to similar studies, the sensitivity for autoantibodies is not very high, which may influence the results of sequence comparison. Since different detection methods and manufacturers vary greatly in antibody measurement, contradictory results could arise(33). In the future, quantitative/semi-quantitative detection methods such as chemiluminescence analysis (CLIA) could be applied to reduce systemic detection error. In addition, both patients and health individual involved in the study were relatively homogenous, and may not reflect real-life condition. A larger sample size and inclusion of patients with a wider range of associated diseases or clinical features could further complement the study.

\section{Conclusion}

In conclusion, detection of aCL IgA, a $32 \mathrm{GPI} \operatorname{IgA}$, aAnxV IgG/M, and aPS/PT IgG/M as biomarker provide additive value in APS diagnosis, especially aCL IgA and aAnxV IgG. Detecting aCL IgA and aAnxV IgM assist in identifying seronegative APS patients. IgG of aANxV or aPS/PT was significantly associated with $\mathrm{LA}$, and IgG aANxV was linked with stroke, which would assist in risk prediction for APS patients in medical practice.

\section{Author Contributions}

All authors were involved in the design of this study. CH, SL, ZX, HY, HJ, and JZ contributed to the collection of blood samples and other experimental procedures. YS and WQ were involved in data collection and pre-processing. CH and SL analyzed the data and wrote the manuscript. JZ, QW, XT, ML, and YZ contributed to the recruitment of patients and evaluation of clinical data.

\section{Conflict of Interest}

All authors declare no conflicts of interest.

\section{Funding}

This study was supported by the National Natural Science Foundation of China (81771780), the National Key Research and Development Program of China (2019YFC0840603, 2017YFC0907601, and 2017YFC0907602), and the CAMS Initiative for Innovative Medicine (2017-I2M-3-001 and 2019-I2M-2-008).

\section{References}

1. MIYAKIS S, LOCKSHIN MD, ATSUMI T, BRANCH DW, BREY RL, CERVERA R, et al. International consensus statement on an update of the classification criteria for definite antiphospholipid syndrome (APS). 2006;4(2):295-306.

2. Hughes GR, Khamashta MA. Seronegative antiphospholipid syndrome. Annals of the rheumatic diseases. 2003;62(12):1127.

3. Pignatelli P, Ettorre E, Menichelli D, Pani A, Violi F, Pastori D. Seronegative antiphospholipid syndrome: refining the value of "non-criteria" antibodies for diagnosis and clinical management. Haematologica. 2020;105(3):562-72.

4. Devreese KM, Pierangeli SS, de Laat B, Tripodi A, Atsumi T, Ortel TL. Testing for antiphospholipid antibodies with solid phase assays: guidance from the SSC of the ISTH. Journal of thrombosis and haemostasis : JTH. 2014;12(5):792-5. 
5. Volkov I, Seguro L, Leon EP, Kovács L, Roggenbuck D, Schierack P, et al. Profiles of criteria and noncriteria anti-phospholipid autoantibodies are associated with clinical phenotypes of the antiphospholipid syndrome. Auto- immunity highlights. 2020;11(1):8.

6. Litvinova E, Darnige L, Kirilovsky A, Burnel Y, de Luna G, Dragon-Durey MA. Prevalence and Significance of Non-conventional Antiphospholipid Antibodies in Patients With Clinical APS Criteria. Frontiers in immunology. 2018;9:2971.

7. Bertolaccini ML, Amengual O, Andreoli L, Atsumi T, Chighizola CB, Forastiero R, et al. 14th International Congress on Antiphospholipid Antibodies Task Force. Report on antiphospholipid syndrome laboratory diagnostics and trends. Autoimmunity reviews. 2014;13(9):917-30.

8. Lakos G, Favaloro EJ, Harris EN, Meroni PL, Tincani A, Wong RC, et al. International consensus guidelines on anticardiolipin and anti- $\beta 2$-glycoprotein I testing: report from the 13th International Congress on Antiphospholipid Antibodies. Arthritis Rheum. 2012;64(1):1-10.

9. Iaccarino L, Ghirardello A, Canova M, Zen M, Bettio S, Nalotto L, et al. Anti-annexins autoantibodies: their role as biomarkers of autoimmune diseases. Autoimmunity reviews. 2011;10(9):553-8.

10. Andree HA, Stuart MC, Hermens WT, Reutelingsperger CP, Hemker HC, Frederik PM, et al. Clustering of lipid-bound annexin V may explain its anticoagulant effect. The Journal of biological chemistry. 1992;267(25):17907-12.

11. Rodríguez-García V, Ioannou Y, Fernández-Nebro A, Isenberg DA, Giles IP. Examining the prevalence of non-criteria anti-phospholipid antibodies in patients with anti-phospholipid syndrome: a systematic review. Rheumatology (Oxford, England). 2015;54(11):2042-50.

12. Singh NK, Yadav DP, Gupta A, Singh U, Godara M. Role of anti-annexin A5 in pathogenesis of hypercoagulable state in patients with antiphospholipid syndrome. International journal of rheumatic diseases. 2013;16(3):325-30.

13. de Laat B, Derksen RH, Mackie IJ, Roest M, Schoormans S, Woodhams BJ, et al. Annexin A5 polymorphism $(-1 \mathrm{C}->\mathrm{T})$ and the presence of anti-annexin $\mathrm{A} 5$ antibodies in the antiphospholipid syndrome. Annals of the rheumatic diseases. 2006;65(11):1468-72.

14. Rand JH, Wu XX, Lapinski R, van Heerde WL, Reutelingsperger CP, Chen PP, et al. Detection of antibody-mediated reduction of annexin A5 anticoagulant activity in plasmas of patients with the antiphospholipid syndrome. Blood. 2004;104(9):2783-90.

15. Ueki H, Mizushina T, Laoharatchatathanin T, Terashima R, Nishimura Y, Rieanrakwong D, et al. Loss of maternal annexin A5 increases the likelihood of placental platelet thrombosis and foetal loss. Scientific reports. 2012;2:827.

16. Ogawa H, Zhao D, Dlott JS, Cameron GS, Yamazaki M, Hata T, et al. Elevated anti-annexin V antibody levels in antiphospholipid syndrome and their involvement in antiphospholipid antibody specificities. American journal of clinical pathology. 2000;114(4):619-28.

17. Arnold J, Holmes Z, Pickering W, Farmer C, Regan L, Cohen H. Anti-beta 2 glycoprotein 1 and antiannexin V antibodies in women with recurrent miscarriage. British journal of haematology. 2001;113(4):911-4.

18. Amengual O, Forastiero R, Sugiura-Ogasawara M, Otomo K, Oku K, Favas C, et al. Evaluation of phosphatidylserine-dependent antiprothrombin antibody testing for the diagnosis of antiphospholipid syndrome: results of an international multicentre study. Lupus. 2017;26(3):266-76.

19. Sciascia S, Sanna G, Murru V, Roccatello D, Khamashta MA, Bertolaccini ML. Anti-prothrombin (aPT) and anti-phosphatidylserine/prothrombin (aPS/PT) antibodies and the risk of thrombosis in the antiphospholipid syndrome. A systematic review. Thrombosis and haemostasis. 2014;111(2):354-64. 
20. Devreese KMJ, Ortel TL, Pengo V, de Laat B. Laboratory criteria for antiphospholipid syndrome: communication from the SSC of the ISTH. Journal of thrombosis and haemostasis : JTH. 2018;16(4):809-13.

21. Atsumi T, Ieko M, Bertolaccini ML, Ichikawa K, Tsutsumi A, Matsuura E, et al. Association of autoantibodies against the phosphatidylserine-prothrombin complex with manifestations of the antiphospholipid syndrome and with the presence of lupus anticoagulant. Arthritis Rheum. 2000;43(9):1982-93.

22. Sciascia S, Radin M, Cecchi I, Rubini E, Scotta A, Rolla R, et al. Reliability of Lupus Anticoagulant and Anti-phosphatidylserine/prothrombin Autoantibodies in Antiphospholipid Syndrome: A Multicenter Study. Frontiers in immunology. 2019;10:376.

23. Pengo V, Ruffatti A, Legnani C, Testa S, Fierro T, Marongiu F, et al. Incidence of a first thromboembolic event in asymptomatic carriers of high-risk antiphospholipid antibody profile: a multicenter prospective study. Blood. 2011;118(17):4714-8.

24. Zhang S, Wu Z, Li J, Wen X, Li L, Zhang W, et al. Evaluation of the clinical relevance of anti-annexin-A5 antibodies in Chinese patients with antiphospholipid syndrome. Clinical rheumatology. 2017;36(2):407-12.

25. Shi H, Zheng H, Yin YF, Hu QY, Teng JL, Sun Y, et al. Antiphosphatidylserine/prothrombin antibodies (aPS/PT) as potential diagnostic markers and risk predictors of venous thrombosis and obstetric complications in antiphospholipid syndrome. Clinical chemistry and laboratory medicine. 2018;56(4):614-24.

26. Zhu L, Li C, Liu N, Yang X, Jia RL, Mu R, et al. Diagnostic value of antibodies to phosphatidylserine/prothrombin complex for antiphospholipid syndrome in Chinese patients. Clinical rheumatology. 2017;36(2):401-6.

27. Liu T, Gu J, Wan L, Hu Q, Teng J, Liu H, et al. "Non-criteria" antiphospholipid antibodies add value to antiphospholipid syndrome diagnoses in a large Chinese cohort. Arthritis research \& therapy. 2020;22(1):33.

28. Hu C, Li X, Zhao J, Wang Q, Li M, Tian X, et al. Immunoglobulin A Isotype of Antiphospholipid Antibodies Does Not Provide Added Value for the Diagnosis of Antiphospholipid Syndrome in a Chinese Population. Frontiers in immunology. 2020;11:568503.

29. Paule R, Morel N, Le Guern V, Fredi M, Coutte L, Belhocine M, et al. Classification of primary antiphospholipid syndrome as systemic lupus erythematosus: Analysis of a cohort of 214 patients. Autoimmunity reviews. 2018;17(9):866-72.

30. Vlagea A, Gil A, Cuesta MV, Arribas F, Diez J, Lavilla P, et al. Antiphosphatidylserine/prothrombin antibodies (aPS/PT) as potential markers of antiphospholipid syndrome. Clinical and applied thrombosis/hemostasis : official journal of the International Academy of Clinical and Applied Thrombosis/Hemostasis. 2013;19(3):289-96.

31. Žigon P, Perdan Pirkmajer K, Tomšič M, Kveder T, Božič B, Sodin Šemrl S, et al. AntiPhosphatidylserine/Prothrombin Antibodies Are Associated with Adverse Pregnancy Outcomes. Journal of immunology research. 2015;2015:975704.

32. Andreoli L, Chighizola CB, Banzato A, Pons-Estel GJ, Ramire de Jesus G, Erkan D. Estimated frequency of antiphospholipid antibodies in patients with pregnancy morbidity, stroke, myocardial infarction, and deep vein thrombosis: a critical review of the literature. Arthritis care \& research. 2013;65(11):1869-73.

33. Devreese KMJ. How to Interpret Antiphospholipid Laboratory Tests. Current rheumatology reports. 2020;22(8):38.

Table 1. Demographic and clinical variables of subjects $(n=312)$.

\begin{tabular}{llllll}
\hline & APS (151) & APS (151) & APS (151) & SLE (71) & SLE (71) \\
\hline & Primary (100) & Primary (100) & Secondary (51) & & \\
Gender (female/male) & $63 / 37$ & $45 / 6$ & $45 / 6$ & $61 / 10$ & $41 / 49$
\end{tabular}




\begin{tabular}{|c|c|c|c|c|c|}
\hline & APS (151) & APS (151) & APS (151) & SLE (71) & SLE $(71$ \\
\hline Mean age (years $\pm \mathrm{SD})$ & $36.3 \pm 12.1$ & $32.9 \pm 10.2$ & $32.9 \pm 10.2$ & $30.1 \pm 8.2$ & $43.4 \pm 12$ \\
\hline \multicolumn{6}{|l|}{ Clinical manifestations } \\
\hline Thrombosis, n (\%) & $80(80.0 \%)$ & $38(74.5 \%)$ & $38(74.5 \%)$ & 0 & NA \\
\hline Pregnancy morbidity, n (\%) & $33(33.0 \%)$ & $16(31.4 \%)$ & $16(31.4 \%)$ & 0 & NA \\
\hline Thrombosis + pregnancy morbidity, n (\%) & $13(13 \%)$ & $3(5.9 \%)$ & $3(5.9 \%)$ & 0 & NA \\
\hline $\mathrm{LA}, \mathrm{n}(\%)$ & $73(73.0 \%)$ & $44(86.3 \%)$ & $44(86.3 \%)$ & $17(23.9 \%)$ & NA \\
\hline History of arterial thrombosis, n (\%) & $43(43.0 \%)$ & $21(41.2 \%)$ & $21(41.2 \%)$ & 0 & NA \\
\hline Stroke, n (\%) & $4(4.0 \%)$ & $2(3.9 \%)$ & $2(3.9 \%)$ & 0 & NA \\
\hline Coronary heart disease, n (\%) & $9(9.0 \%)$ & $2(3.8 \%)$ & $2(3.8 \%)$ & 0 & NA \\
\hline Eye involvement, $\mathrm{n}(\%)$ & $3(3.0 \%)$ & $1(2.0 \%)$ & $1(2.0 \%)$ & & \\
\hline Lower limb artery occlusion, n (\%) & $1(1.0 \%)$ & 0 & 0 & 0 & NA \\
\hline History of venous thrombosis, n (\%) & $47(47.0 \%)$ & $24(47.1 \%)$ & $24(47.1 \%)$ & 0 & NA \\
\hline Deep vein thrombosis, n (\%) & $19(19.0 \%)$ & $7(13.7 \%)$ & $7(13.7 \%)$ & 0 & NA \\
\hline Pulmonary embolism, n (\%) & $19(19.0 \%)$ & $2(3.9 \%)$ & $2(3.9 \%)$ & 0 & NA \\
\hline Upper limb vein thrombosis, n (\%) & 0 & $1(2.0 \%)$ & $1(2.0 \%)$ & 0 & NA \\
\hline Renal vein thrombosis, n (\%) & $1(1.0 \%)$ & 0 & 0 & 0 & NA \\
\hline Portal vein thrombosis, n (\%) & $4(4.0 \%)$ & $1(2.0 \%)$ & $1(2.0 \%)$ & 0 & NA \\
\hline Cerebral venous and sinus thrombosis, n (\%) & $3(3.0 \%)$ & $1(2.0 \%)$ & $1(2.0 \%)$ & 0 & NA \\
\hline Central retinal venous occlusion, $\mathrm{n}(\%)$ & $1(1.0 \%)$ & 0 & 0 & 0 & NA \\
\hline Microangiopathy, n (\%) & $57(57 \%)$ & $24(47.1 \%)$ & $24(47.1 \%)$ & 0 & NA \\
\hline $\mathrm{TP}, \mathrm{n}(\%)$ & $38(38 \%)$ & $* 28(54.9 \%)$ & $* 28(54.9 \%)$ & $21(29.6 \%)$ & NA \\
\hline Heat valve disease, $\mathrm{n}(\%)$ & 0 & $6(11.8 \%)$ & $6(11.8 \%)$ & 0 & NA \\
\hline Non-stroke CNS manifestations, n (\%) & $4(4.0 \%)$ & $4(7.8 \%)$ & $4(7.8 \%)$ & 0 & NA \\
\hline Antiphospholipid syndrome nephropathy, n (\%) & $6(6.0 \%)$ & $2(3.8 \%)$ & $2(3.8 \%)$ & 0 & NA \\
\hline Autoimmune hemolytic anemia, n (\%) & $1(1.0 \%)$ & $5(9.8 \%)$ & $5(9.8 \%)$ & 0 & NA \\
\hline Thrombotic Microangiopathy, n (\%) & 0 & $1(2.0 \%)$ & $1(2.0 \%)$ & 0 & NA \\
\hline Hemolytic uremic syndrome, n (\%) & $1(1.0 \%)$ & 0 & 0 & 0 & NA \\
\hline History of adverse pregnancy, n (\%) & $37(37 \%)$ & $20(39.2 \%)$ & $20(39.2 \%)$ & $4(5.6 \%)$ & NA \\
\hline Early fetal loss (<10 weeks), n (\%) & $12(12.0 \%)$ & $8(15.7 \%)$ & $8(15.7 \%)$ & $4(5.6 \%)$ & NA \\
\hline Late fetal loss (10-28 weeks), n (\%) & $19(19.0 \%)$ & $12(23.5 \%)$ & $12(23.5 \%)$ & 0 & NA \\
\hline Placental insufficiency, n (\%) & $14(14.0 \%)$ & $7(13.7 \%)$ & $7(13.7 \%)$ & 0 & NA \\
\hline
\end{tabular}

${ }^{*} P=0.048$, significant different from primary APS

Table 2. The predictive value of different aPLs in APS diagnosis

\begin{tabular}{|c|c|c|c|c|c|c|c|}
\hline & $\begin{array}{l}\text { Sensitivity } \\
(\%)\end{array}$ & $\begin{array}{l}\text { Specificity } \\
(\%)\end{array}$ & $\begin{array}{l}\text { Accuracy } \\
(\%)\end{array}$ & $\begin{array}{l}\text { Youden } \\
\text { Index }\end{array}$ & PPV (\%) & NPV $(\%)$ & $\begin{array}{l}\text { OR }(95 \% \\
\text { CI })\end{array}$ \\
\hline $\mathrm{aCL} \operatorname{IgG}$ & 37.09 & 100.00 & 60.58 & 0.371 & 100.00 & 48.65 & {$[?]$} \\
\hline aCL IgM & 8.61 & 97.78 & 41.90 & 0.064 & 86.67 & 38.94 & $\begin{array}{l}4.15(0.91- \\
18.81)\end{array}$ \\
\hline $\begin{array}{l}\text { aCL IgG } \\
\text { or IgM }\end{array}$ & 41.06 & 97.78 & 62.24 & 0.389 & 96.88 & 49.72 & $\begin{array}{l}30.65(7.27- \\
129.20)\end{array}$ \\
\hline $\begin{array}{l}\text { aß2GpI } \\
\text { IgG }\end{array}$ & 23.18 & 100.00 & 51.86 & 0.232 & 100.00 & 43.69 & {$[?]$} \\
\hline $\begin{array}{l}\text { a } \beta 2 \mathrm{GpI} \\
\text { IgM }\end{array}$ & 7.95 & 98.89 & 41.91 & 0.068 & 92.31 & 39.04 & $\begin{array}{l}7.68(0.98- \\
60.12)\end{array}$ \\
\hline
\end{tabular}




\begin{tabular}{|c|c|c|c|c|c|c|c|}
\hline & $\begin{array}{l}\text { Sensitivity } \\
(\%)\end{array}$ & $\begin{array}{l}\text { Specificity } \\
(\%)\end{array}$ & $\begin{array}{l}\text { Accuracy } \\
(\%)\end{array}$ & $\begin{array}{l}\text { Youden } \\
\text { Index }\end{array}$ & PPV (\%) & $\operatorname{NPV}(\%)$ & $\begin{array}{l}\text { OR }(95 \% \\
\text { CI })\end{array}$ \\
\hline $\begin{array}{l}\text { a } \beta 2 \mathrm{GpI} \\
\text { IgG or } \\
\text { IgM }\end{array}$ & 29.14 & 98.89 & 55.19 & 0.28 & 97.78 & 45.41 & $\begin{array}{l}36.60(4.94- \\
270.96)\end{array}$ \\
\hline $\begin{array}{l}\text { aCL or } \\
\text { aB2GpI } \\
\text { IgG or } \\
\text { IgM }\end{array}$ & 43.05 & 97.78 & 63.48 & 0.408 & 97.01 & 50.57 & $\begin{array}{l}33.26(7.89- \\
140.10)\end{array}$ \\
\hline aCL IgA & 30.46 & 92.22 & 53.53 & 1.2268 & 86.79 & 44.15 & $\begin{array}{l}5.19(2.23- \\
12.10)\end{array}$ \\
\hline $\begin{array}{l}\text { a } \beta 2 \mathrm{GpI} \\
\text { IgA }\end{array}$ & 6.62 & 98.89 & 41.08 & 1.0551 & 90.91 & 38.70 & $\begin{array}{l}6.31(0.79- \\
50.16)\end{array}$ \\
\hline $\begin{array}{l}\text { aCL IgG } \\
\text { or IgM or } \\
\text { IgA }\end{array}$ & 51.66 & 91.11 & 66.39 & 1.4277 & 90.70 & 52.90 & $\begin{array}{l}10.95(4.96- \\
24.21)\end{array}$ \\
\hline $\begin{array}{l}\text { a } \beta 2 \mathrm{GpI} \\
\text { IgG or } \\
\text { IgM or } \\
\text { IgA }\end{array}$ & 31.79 & 97.78 & 56.43 & 1.2957 & 96.00 & 46.07 & $\begin{array}{l}20.51(4.85- \\
86.79)\end{array}$ \\
\hline $\begin{array}{l}\mathrm{aCL} \text { or } \\
\mathrm{aB} 2 \mathrm{GpI} \\
\mathrm{IgG} \text { or } \\
\text { IgM or } \\
\text { IgA }\end{array}$ & 53.64 & 91.11 & 67.63 & & 91.01 & 53.95 & $\begin{array}{l}11.86(5.37- \\
26.22)\end{array}$ \\
\hline $\mathrm{P} 1$ & $<0.001$ & 0.031 & 0.052 & & & & \\
\hline P2 & 0.125 & 1.000 & 0.375 & & & & \\
\hline P3 & $<0.001$ & 0.031 & 0.052 & & & & \\
\hline $\begin{array}{l}\text { aPS/PT } \\
\mathrm{IgG}\end{array}$ & 18.54 & 96.67 & 47.72 & 0.152 & 90.32 & 41.43 & $\begin{array}{l}6.60(1.95- \\
22.40)\end{array}$ \\
\hline $\begin{array}{l}\text { aPS/PT } \\
\text { IgM }\end{array}$ & 7.28 & 98.89 & 41.49 & 0.062 & 91.67 & 38.86 & $\begin{array}{l}6.99(0.89- \\
55.10)\end{array}$ \\
\hline $\begin{array}{l}\text { aPS/PT } \\
\text { IgG or } \\
\text { IgM }\end{array}$ & 24.50 & 95.56 & 51.03 & 0.201 & 90.24 & 43.00 & $\begin{array}{l}6.99(2.40- \\
20.32)\end{array}$ \\
\hline $\begin{array}{l}\text { aCL, } \\
\text { aB2GpI, } \\
\text { or } \\
\text { aPS/PT } \\
\text { IgG or } \\
\text { IgM }\end{array}$ & 45.70 & 94.44 & 63.90 & 0.401 & 93.24 & 50.90 & $\begin{array}{l}14.31(5.49- \\
37.25)\end{array}$ \\
\hline $\mathrm{P} 1{ }^{\prime}$ & $<0.001$ & 0.625 & $<0.001$ & & & & \\
\hline $\begin{array}{l}\text { P2' } \\
\text { P3 }\end{array}$ & $\begin{array}{l}0.167 \\
0.125\end{array}$ & $\begin{array}{l}0.375 \\
0.250\end{array}$ & $\begin{array}{l}0.064 \\
1.000\end{array}$ & & & & \\
\hline $\begin{array}{l}\text { P3 } \\
\text { aAnxV } \\
\text { IgG }\end{array}$ & $\begin{array}{l}0.125 \\
30.46\end{array}$ & $\begin{array}{l}0.250 \\
100.00\end{array}$ & $\begin{array}{l}1.000 \\
56.43\end{array}$ & 0.305 & 100.00 & 46.15 & {$[?]$} \\
\hline $\begin{array}{l}\text { aAnxV } \\
\text { IgM }\end{array}$ & 16.56 & 96.67 & 46.47 & 0.133 & 89.29 & 40.85 & $\begin{array}{l}5.75(1.69- \\
19.70)\end{array}$ \\
\hline $\begin{array}{l}\text { aAnxV } \\
\text { IgG or } \\
\text { IgM }\end{array}$ & 39.07 & 96.67 & 60.58 & 0.358 & 95.16 & 48.60 & $\begin{array}{l}18.60(5.62- \\
61.53)\end{array}$ \\
\hline
\end{tabular}




\begin{tabular}{llllllll}
\hline & $\begin{array}{l}\text { Sensitivity } \\
(\%)\end{array}$ & $\begin{array}{l}\text { Specificity } \\
(\%)\end{array}$ & $\begin{array}{l}\text { Accuracy } \\
(\%)\end{array}$ & $\begin{array}{l}\text { Youden } \\
\text { Index }\end{array}$ & PPV (\%) & NPV (\%) & $\begin{array}{l}\text { OR }(95 \% \\
\text { CI })\end{array}$ \\
\hline $\begin{array}{l}\text { aCL, } \\
\text { aß2GpI, or }\end{array}$ & 47.68 & 96.67 & 65.98 & 0.444 & 96.00 & 52.41 & $\begin{array}{l}26.43(8.01- \\
87.26)\end{array}$ \\
aAnxV & & & & & & \\
IgG or & & & & & & \\
IgM & & & & & & \\
P1" & 0.648 & 1.000 & 0.503 & & & \\
P2" & $<\mathbf{0 . 0 0 1}$ & 0.500 & $\mathbf{0 . 0 0 7}$ & & & \\
P3" & $\mathbf{0 . 0 1 6}$ & 1.000 & 0.070 & & & & \\
\hline
\end{tabular}

PPV, positive predictive value; NPV, negative predictive value; OR, odds ratio; CI, confidence interval.Pvalues of sensitivity, specificity, and accuracy are calculated with McNemar test.

$\mathrm{P}_{1}$ : Comparison of result of aCl IgG or IgM or IgA to aCL IgG or IgM; $\mathrm{P}_{2}$ : Comparison of result of a $32 \mathrm{GpI}$ $\operatorname{IgG}$ or IgM or IgA to a $32 \mathrm{GpI} \operatorname{IgG}$ or IgM; $\mathrm{P}_{3}$ : Comparison of result of aCL or a $\beta 2 \mathrm{GpI} \operatorname{IgG}$ or IgM or $\operatorname{IgA}$ to aCL or a $22 \mathrm{GpI} \operatorname{IgG}$ or IgM; $\mathrm{P}_{1}$ : Comparison of result of $\mathrm{aPS} / \mathrm{PT} \operatorname{IgG}$ or IgM to aCL IgG or IgM; $\mathrm{P}_{2}$,: Comparison of result of aPS/PT IgG or IgM to a $\beta 2 \mathrm{GpI}$ IgG or IgM;P ${ }_{3}$ : Comparison of result of aCL, aß2GpI, or aPS/PT IgG or IgM to aCL or a $\beta 2 \mathrm{GpI}$ IgG or IgM; $\mathrm{P}_{1}$ ": Comparison of result of aAnxV IgG or IgM to aCL IgG or IgM; $\mathrm{P}_{2}$ ": Comparison of result of aAnxV IgG or IgM to a $\beta 2 \mathrm{GpI} \operatorname{IgG}$ or IgM; $\mathrm{P}_{3}$ ": Comparison of result of aCL, aB2GpI, or aAnxV IgG or IgM to aCL or a $32 \mathrm{GpI}$ IgG or IgM;Odds ratios (ORs) with $95 \%$ confidence intervals (CIs) are shown.

Table 3 Correlations between different aPLs and clinical manifestations among APS patients $(\mathrm{n}=151)$

\begin{tabular}{|c|c|c|c|c|c|}
\hline & Thrombosis & Arterial thrombosis & Venous thrombosis & Venous thrombosis & Pregnancy morbidity \\
\hline $\mathrm{aCL} \operatorname{IgG}$ & $1.3(0.6-3.1)$ & $1.6(0.8-3.2)$ & $0.8(0.4-1.6)$ & $1.2(0.6-2.7)$ & $1.2(0.6-2.7)$ \\
\hline $\mathrm{aCL}$ IgM & $0.6(0.2-1.9)$ & $0.8(0.3-2.7)$ & $0.7(0.2-2.3)$ & $1.0(0.3-3.5)$ & $1.0(0.3-3.5)$ \\
\hline aCL IgA & $0.6(0.2-1.2)$ & $0.8(0.4-1.5)$ & $0.8(0.4-1.5)$ & $0.9(0.4-1.9)$ & $0.9(0.4-1.9)$ \\
\hline aß2GPI IgG & $1.7(0.6-5.0)$ & $1.6(0.8-3.4)$ & $1.0(0.5-2.1)$ & $1.1(0.5-2.9)$ & $1.1(0.5-2.9)$ \\
\hline aß2GPI IgM & $1.3(0.3-6.5)$ & $0.4(0.1-1.6)$ & $2.5(0.7-8.6)$ & $0.9(0.2-4.2)$ & $0.9(0.2-4.2)$ \\
\hline a $\beta 2$ GPI IgA & $1.1(0.2-5.2)$ & $0.9(0.2-3.3)$ & $1.8(0.5-6.7)$ & $1.0(1.0-1.1)$ & $1.0(1.0-1.1)$ \\
\hline aPS/PT IgG & $1.3(0.4-3.6)$ & $1.7(0.8-4.0)$ & $0.7(0.3-1.6)$ & $1.3(0.5-3.4)$ & $1.3(0.5-3.4)$ \\
\hline aPS/PT IgM & $1.2(0.2-5.8)$ & $0.8(0.2-2.7)$ & $2.1(0.6-7.6)$ & $0.4(0.1-2.0)$ & $0.4(0.1-2.0)$ \\
\hline aAnxV IgG & $1.7(0.7-4.3)$ & $1.8(0.9-3.6)$ & $0.8(0.4-1.7)$ & $1.0(0.4-2.3)$ & $1.0(0.4-2.3)$ \\
\hline aAnxV IgM & $1.1(0.4-3.1)$ & $1.3(0.6-3.1)$ & $0.7(0.3-1.8)$ & $1.0(0.3-2.6)$ & $1.0(0.3-2.6)$ \\
\hline
\end{tabular}

Odds ratios (ORs) with $95 \%$ confidence intervals (CIs) are shown. ${ }^{*} P<0.05$ )

Figure 1. Comparison of receiver operating characteristic (ROC) curves and area under the curve (AUC). ORs with 95\% CIs are shown. 

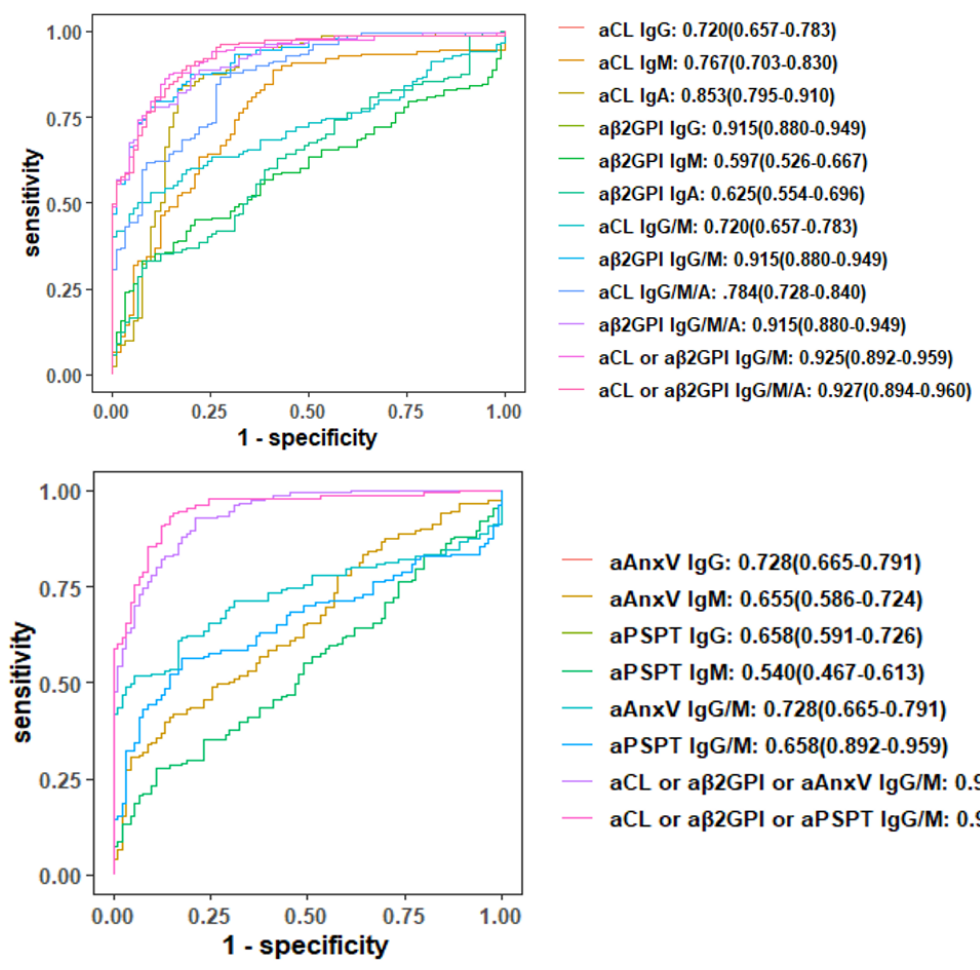

- aAnxV IgG: $0.728(0.665-0.791)$

- aAnxV IgM: 0.655(0.586-0.724)

- aPSPT IgG: $0.658(0.591-0.726)$

— aPSPT IgM: 0.540(0.467-0.613)

— aAnxV IgG/M: 0.728(0.665-0.791)

— aPSPT IgG/M: 0.658(0.892-0.959)

- aCL or a $32 \mathrm{GPI}$ or aAnxV IgG/M: 0.936(0.907-0.965)

- aCL or aß2GPI or aPSPT IgG/M: 0.951(0.924-0.977)

Figure2. Venn diagram of aPLs cross positivity analysis in APS group $(n=151)$

a)

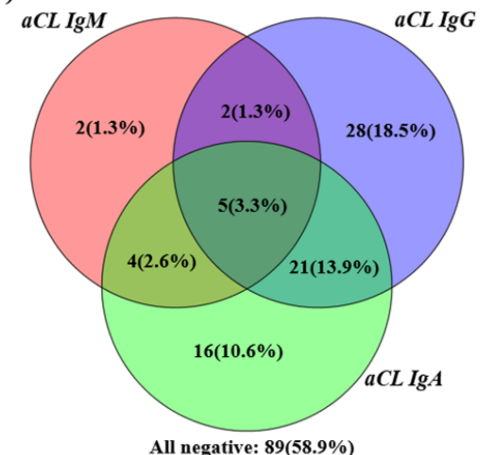

c)

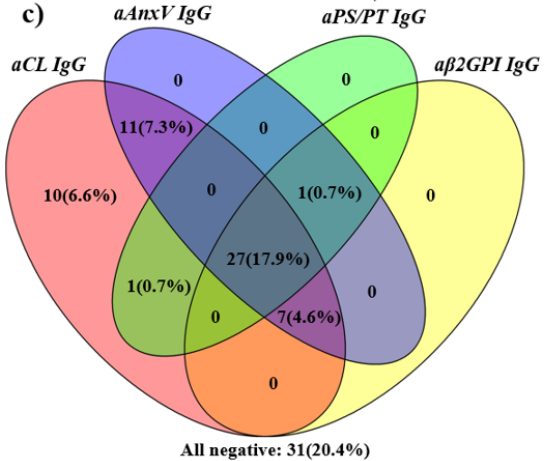

b)
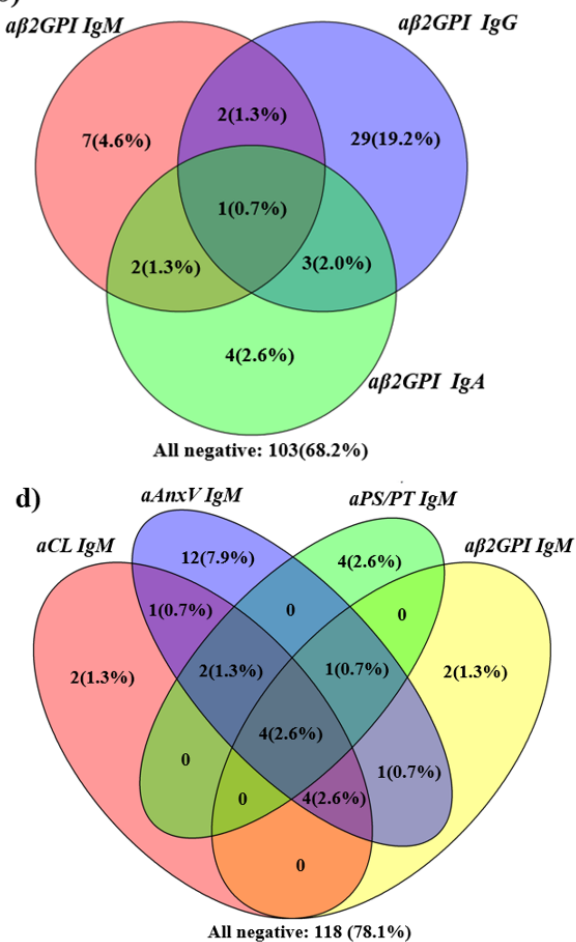
a) cross positivity for aCL; b) cross positivity for aß2GpI; c) cross positivity for IgG; d) cross positivity for $\operatorname{IgM}$

Figure 3. Distribution of IgG and IgM for four antibodies among different patient groups
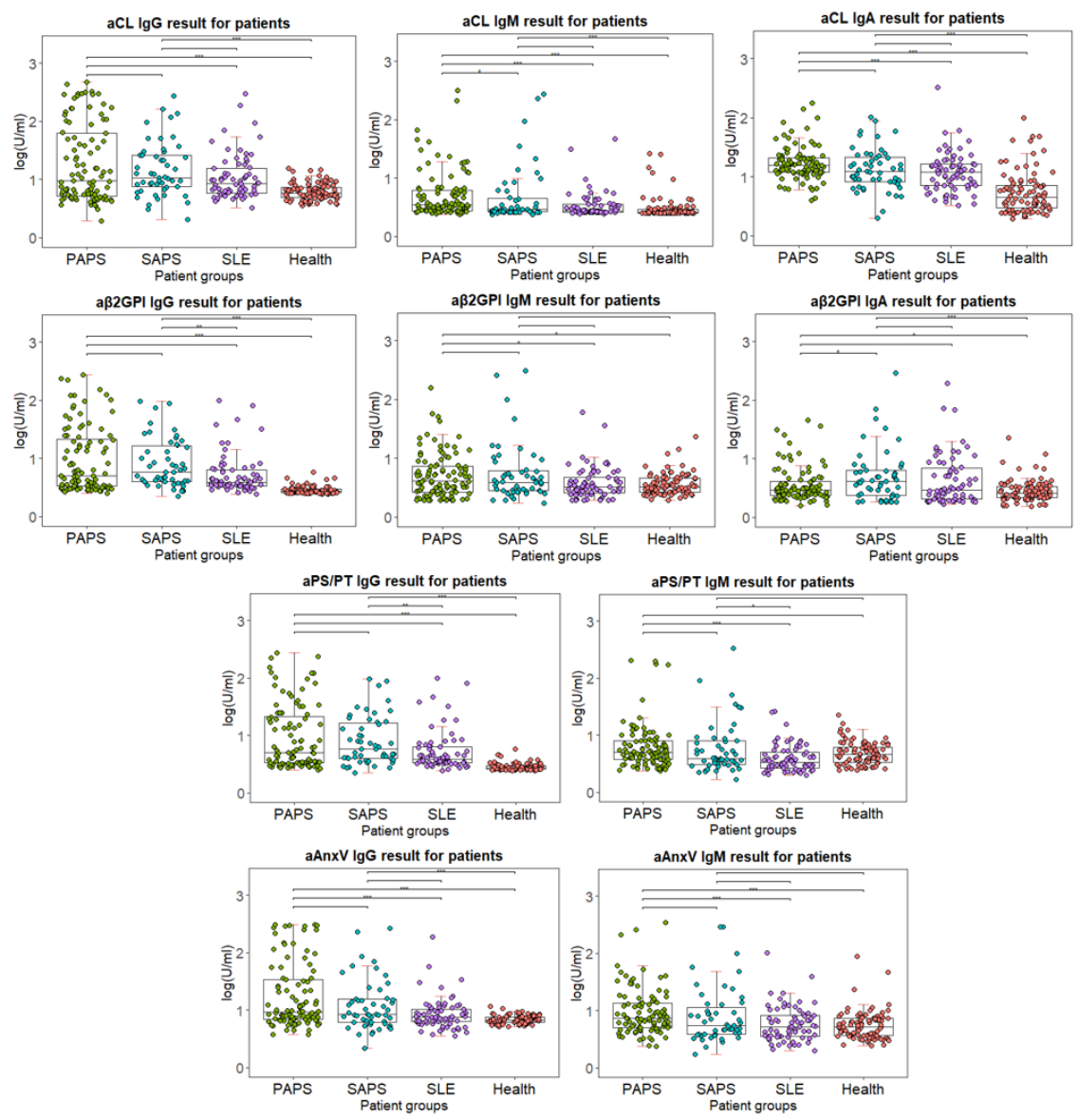

Test results are calculated using $\lg ($ test value +2 ), in order for the value to be shown in positive number. Wilcox's test is conducted comparing primary or secondary APS results to other patient groups. ${ }^{*} \mathrm{p}<0.05$. ${ }^{* *} \mathrm{p}<0.01,{ }^{* * *} \mathrm{p}<0.001$, NS: not significant. 